\title{
The Role of Blue/Green Laser Systems in Strategic Submarine Communications
}

\author{
THOMAS F. WIENER, MEMBER, IEEE, AND SHERMAN KARP, MEMBER, IEEE
}

\begin{abstract}
A continuing interest exists in the Department of Defense to stress technology wherever it may result in the improvement of a military system. One important area where this is currently occurring is in laser communications. Specifically, an extensive effort is underway to exploit the "Blue/Green" transmission window in the ocean and develop a strategic laser communication system operating between an orbiting satellite and a submerged submarine. In this paper we discuss some of the operational motivations for such a system together with a generic system design.
\end{abstract}

\section{INTRODUCTION}

A LL organized operations require communications. In order for disparate parts of an organization to act in concert, direction must be communicated from a central command. The particular communications of interest in this paper are those between the National Command Authority (NCA) and ballistic missile submarines (SSBN's) at sea. This is a vital portion of this country's strategic communications network, and as such, must always be improved whenever the technology permits. In this paper we will briefly discuss the status of current techniques and one of the candidates presently being studied as a potential future improvement. We will then perform a first level system analysis of this system to highlight some of the peculiar problems associated with it and the emerging design philosophy.

\section{OPERATIONAL CONSIDERATIONS}

The primary objectives of the communications system between NCA and the SSBN's is to deliver the messages of required length without compromising the position of the SSBN's. The SSBN's at sea are the most survivable leg of the nations's nuclear deterrent triad. (The other two legs of the triad are the land-based ballistic missiles and the air-breathing bombers and, in the future, cruise missiles.) The primary objective of the SSBN on patrol is to remain undetected, thus assuring its survivaibility. It is also required to receive communications signals at all times, a requirement with constraints at variance with the objective or remaining undetected.

The normal means of communicating with SSNB's is through VLF radio which sends encrypted teletype. There are large shore transmitters on each coast of the United States for this purpose. In addition, signals are sometimes sent from airplanes.

Since VLF electromagnetic radiation is attenuated rapidly by seawater, the submarine's receiving antenna must be quite near the surface. There are two types of antennas in general use today: the buoyant or floating wire and the loop. The loop

Manuscript received January $15,1980$.

The authors are with DARPA/STO, Arlington, VA 22209. may be carried on a retractible mast in the submarine's sail or it may be towed behind the submarine on a cable which also carries the received signal down to the submarine.

There must be about $100 \mathrm{~m}$ of floating wire at the surface; therefore, the deeper a submarine wishes to be, the more wire must be streamed. Also, if a submarine has a given length of wire streamed to maintain $100 \mathrm{~m}$ on the surface, an increase in speed must be accompanied by. a decrease in depth or an increase in amount of wire streamed in order to maintain 100 $m$ on the surface. In addition to the speed and depth restrictions on the submarine which are necessary to keep an adequate amount of the wire on the surface, the wire must be lined up with the (great circle) azimuth to the transmitting station. To the extent that the submarine's course does not coincide with this azimuth or its reciprocal, more wire must be kept on the surface to provide the required projected length. And, of course, more wire on the surface means more wire streamed or a shallower submarine depth or a slower submarine speed.

Use of the mast-mounted loop antenna requires the submarine to be very near the surface. With the mast extended and the loop just below the surface, the submarine is vulnerable to collisions with surface ships, and is severely limited in allowable speed. (Exceeding the speed limit causes the mast to bend.) Course is not restricted because the antenna is a pair of crossed loops, and the combination can be electronically steered. With the towed buoy, the submarine is least limited in depth, and course is still unrestricted. A similar speed limit applies, however, to avoid exceeding the breaking strength of the tow cable, and to allow the buoy to remain just under the surface.

In summary, use of VLF radio to communicate with the submarine imposes operational restrictions on speed, depth, and course. In addition, while enemy surveillance systems do not now pose any threat to the survivability of U.S. SSBN's because of the presence of an antenna at or near the surface, prudence dictates that efforts be made to reduce observables as much as possible.

The ideal communications system would allow receipt of messages by a submarine at any depth or speed on any course. In addition, it should not be necessary to have an antenna which extends beyond the normal streamlined envelope of the submarine. Finally, or perhaps primarily, the system should be able to deliver a message of the required length in the alloted time.

An attractive alternative to RF communication is optical communications. Blue light (about $625 \mathrm{THz}$ ) penetrates water better than any other frequency electromagnetic radiation except for frequencies below $10 \mathrm{~Hz}$. With sufficient power den- 
sity on the surface, information can be detected by a receiver at any depth.

The conceptual system consists of a light source in space, projecting pulsed optical power onto the ocean surface in a spot of a specified area, typically as much as tens to thousands of kilometers in diameter. To reach the submerged receiver, the light must travel through the transmission channel consiting of space, the atmosphere (including clear air and clouds), and water. The detector with a large area is mounted on the top of the submarine sail, and has a narrow bandpass filter. The acceptance angle or field of view should be large enough to capture most of the energy in the down-welling beam.

The operational requirements for our conceptual system are to send a message of a specified length to every receiver in a specified area within a specified time. This is done by positioning the spot of light successively at new positions until the entire area has been covered.

Some qualitative tradeoffs in system performance are immediately suggested. For given channel and receiver characteristics, reducing the spot size increases the depth that can be reached at the expense of increasing the length of time required to cover the area. Increased energy per pulse can be used either to increase the depth reached or, by increasing the spot size, to reduce the time required to cover the area.

The transmission channel is significantly different from the typical RF or microwave channel because there are significant variations in it over short periods of time and over short distances. For example, there may be a region where clouds are present over the eastern half, but the sky is clear over the west. ern half. Variations in water diffuse attenuation coefficient by as much as a factor of three are not uncommon. This variation is responded to by adjusting the spot size adaptively to maintain constant the depth at which the minimum acceptable signal-to-noise ratio is reached.

Clearly, there will be some channel conditions for which the desired depth cannot be reached or, alternatively, for which the entire area cannot be covered in the allotted time. In such cases, either the system is unavailable or further depth restriction must be placed on the submarine.

\section{SYSTEM CONSIDERATION}

It is clear from the previous discussion that a compromise must be made between the availability of the system and its operational capabilities. Thus, as we attempt to operate at greater depths and cover more area per unit time, less margin remains to combat those deleterious channel effects which have attendant large losses. Consequently, as a design philosophy, we attempt to generate as much system margin as possible so that provision can be made for such compromise.

This motivates the need to build as sensitive a receiver as possible, which includes large collecting areas $A$, proper filters, and optimized signal design.

Experiments have shown that light from a point source, after passing through a few scattering lengths in water, will subtend a solid angle to the receiver $\left(\Omega_{\mathrm{fov}}\right)$ of approximately 1 sr about the zenith [1]. This has two implications. First, it will be difficult to spatially discriminate between the source and the sun since both will appear at the same location. Conse- quently, frequency filtering will be very important, but will be aggravated by the large field of view required. (Efforts are currently underway to develop narrow-band, wide field of view filters.) Second, we see that a field of view of 1 sr implies a spatially incoherent signal [2] $\left(\left(A / \lambda^{2}\right)\right.$ spatial degrees of freedom) so that we can only seriously consider a noncoherent communication system. Such systems have been studied in great detail [2], [3].

A further complication to the design problem results from the need to penetrate clouds. Although clouds have low absorption losses, the high degree of multipath scattering causes pulse stretching on the order of tens to hundreds of microseconds, thereby limiting the coherence bandwidth to 10$100 \mathrm{kHz}$. (The Doppler spread is also of the same order.) The natural signaling element would therefore be a pulse 10-100 $\mu \mathrm{s}$ in duration $\Delta T$, with an optical bandwidth $B$ of $10-100 \mathrm{kHz}$ satisfying $\Delta T B \sim 1$. This would then define a degree of freedom to which we could assign the equipartition energy [2]

$$
\lambda^{2} N(f)=\frac{h f}{e^{\frac{h f}{k T}}-1}=N_{o b}
$$

where $N(f)$ is the spectral radiance. At B-G frequencies $f=$ $6 \times 10^{14} \mathrm{~Hz}$, the sun temperature $T=6000 \mathrm{~K}$ and (1) yields

$$
N_{o b}=3.34 \times 10^{-21} \text {. }
$$

If $B_{s}$ is the system bandwidth and $P$ is the pulse rate, then

$$
M=\frac{B_{s}}{B} \cdot \frac{1}{P \Delta T}
$$

is the alphabet size and $R=P \log _{2} M$ is the data rate. The system would operate by transmitting $P$ pulses per second, with each pulse located in one of $(1 / P \Delta T)$ time slots $\Delta T$ seconds wide and one of $B_{s} / B$ frequency bins $B$ cycles wide.

In the foreseeable future, it will not be possible to construct multiple frequency bins nor can we realistically cosider optical filters with less than $10^{9} \mathrm{~Hz}$ bandwidths. Thus, we must constrain our alphabet size to $M=(1 / P \Delta T)$ and accept an increased noise energy of $N_{o b}\left(B_{s} \Delta T\right)$ where $B_{s} \Delta T \cong 10^{4}-$ $10^{6}$. Finally, we must multiply the noise by the number of spatial degrees of freedom $\Omega_{\mathrm{fov}} A / \lambda^{2}$.

We take $E$ to be the energy in an optical pulse and $\eta$ to be the detector quantum efficiency. We further define

$$
\begin{aligned}
& K_{s}=\frac{\eta E L L^{\prime}}{h f} \\
& K_{n}=\frac{\eta}{h f} N_{o b}\left(B_{s} \Delta T\right) \Omega_{f o v} \frac{A}{\lambda^{2}} L=\frac{\eta}{h f} N(f) A B_{s} \Delta T \Omega_{f o v} L
\end{aligned}
$$

where $K_{s}$ are the signal photoelectrons produced at the detector, $K_{n}$ are the noise photoelectrons produced, $L$ is the attenuation common to signal and noise, and $L^{\prime}$ is the additional attenuation experienced only by the signal. The word error probability for this $M$-ary system is determined by Poisson 
statistics [4] and has been computed to be [5]

$$
\begin{aligned}
P_{E}= & 1-\sum_{x=1}^{\infty}\left\{\frac{\left(K_{s}+K_{n}\right)^{x}}{x !} \exp \left[-\left(K_{s}+K_{n}\right)\right]\right. \\
& \left.\cdot\left[\sum_{i=1}^{x-1} \frac{K_{n}^{i}}{i !} e^{-K_{n}}\right]^{M-1}\left[\frac{(1+a)^{M}-1}{M a}\right]\right\} \\
& -\frac{1}{M} \exp \left[-\left(K_{s}+K_{n}\right)\right]
\end{aligned}
$$

where

$$
a=\frac{K_{n}^{x}}{x ! \sum_{i=1}^{x-1} \frac{K_{n}{ }^{i}}{i !}}
$$

when a maximum likelihood decision is made on the output of all $M$ time intervals. A representative set of curves is shown in Fig. 1 for $K_{s}=20$. Notice that performance is strongly dependent on $K_{n}$. This dependence can be quantified in the following manner. First we see that the signal-to-noise ratio of this system is

$$
\frac{S}{N}=\frac{K_{s}^{2}}{K_{s}+K_{n}}
$$

where we assume that receiver noise can be eliminated. Next we set $M=2$ and plot $P_{E}$ versus $K_{n}$ for various values of $S / N$ (Fig. 2). Notice the transition from Poisson statistics to Gaussian statistics as the central limit theorem starts to apply. This transition occurs for $K_{n} \sim 1$ and can alter the relationship between $S / N$ and $P_{E}$ by several decibels. In addition to the fact that $P_{E}$ decreases as $K_{n}$ increases for the same $S / N$, we need to increase $K_{S}$ to maintain $S / N$ constant.

The signal needed is

$$
\begin{aligned}
K_{s} & =\frac{1}{2} \frac{S}{N}\left\{1+\sqrt{1+4 K_{n} /(S / N)}\right\} \\
& \approx \sqrt{\frac{S}{N} K_{n}: \text { background limited }} \\
& \approx \frac{S}{N}: \text { quantum limited. }
\end{aligned}
$$

As an example, consider the case where $K_{n}=0.01$, and hence where $K_{s}=(S / N)$. If we wish to transmit at $10^{3} \mathrm{bits} / \mathrm{s}$ using $70 \mu$ s slots, we must keep $M$ less than 93 and the pulse rate $P$ at $1000 / \log _{2} M$. For a word error $P_{E}$, the corresponding bit error is [6] $P_{E} / 2$ so that a bit error rate of say $10^{-6}$ requires a word error no greater than $2 \times 10^{-6}$. From Fig. 1 we see that this requires $K_{s}=20$ for $M=93$ or

$$
\begin{aligned}
\frac{K_{s}}{\log _{2} M} & =\frac{20}{6.5} \\
& =3.08 \text { photoelectrons/bit. }
\end{aligned}
$$

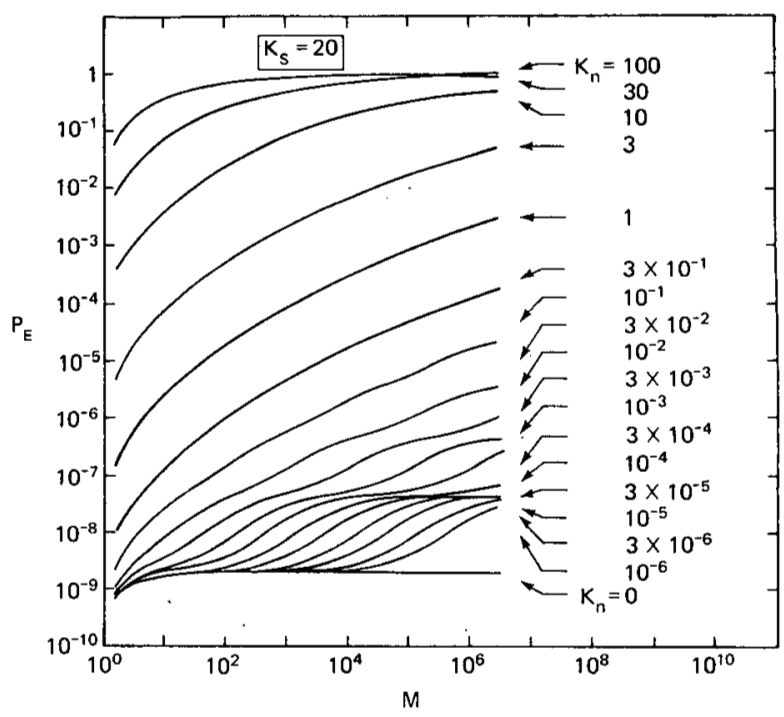

Fig. 1. Error probabilities for $K_{n}$ fixed as a function of $M$.

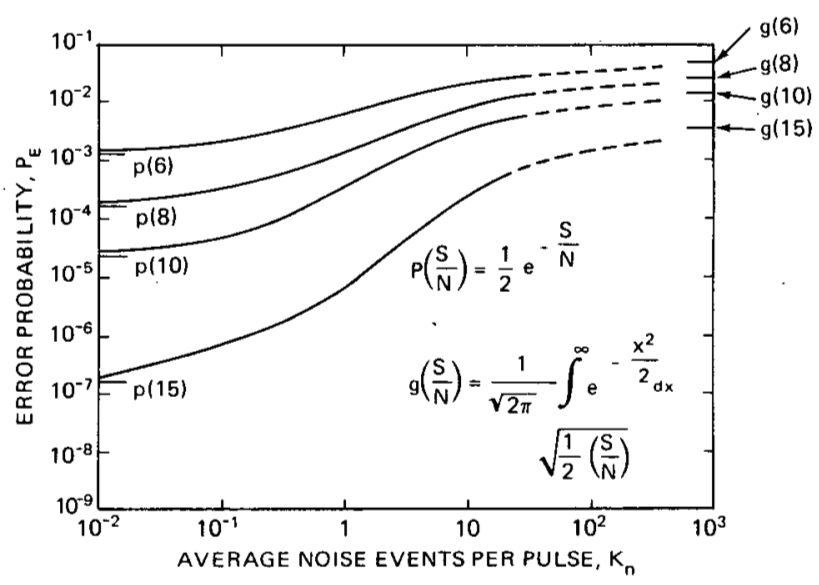

Fig. 2. Error probability versus normalized noise energy $K_{n}$ for fixed values of $S / N=K_{s}{ }^{2} /\left(K_{s}+K_{n}\right)$.

We can also estimate the capacity of this system by recognizing that this is an $M$-ary symmetric channel which yields a capacity of [7]

$$
\begin{aligned}
C= & \log _{2} M+P_{E} \log _{2}\left(P_{E} / M-1\right) \\
& +\left(1-P_{E}\right) \log _{2}\left(1-P_{E}\right) \text { bits/symbol }
\end{aligned}
$$

and an information rate $H$ of

$$
H=\frac{C}{M \Delta T} \text { bits } / \mathrm{s} .
$$

Since $C$ is a function of both $K_{s}$ and $M$, a fixed rate may be achieved with various combinations. These can be obtained as the intersection of $C\left(K_{s}, K_{n}, M\right)$ with $H M \Delta T$ as a function of $M$. Since we desire the most sensitive system, we should choose the combination which minimizes $K_{s} / C$ [8]. In Table I we have tabulated $K_{s}, M, K_{s} / C$ for $H=10^{3}$ and $\Delta T=7 \times$ $10^{-5} \mathrm{~s}$. The minimum value of $K_{s} / C, \beta$, is 0.484 when $M=42$. Although this is $8.03 \mathrm{~dB}$ lower than our example, $P_{E}$ is $2.4 \times$ $10^{-1}$ so that extensive coding would be necessary to achieve the same performance. In Fig. 3 we plot $\beta$ as a function of 
TABLE I

MINIMIZATION OF THE ENERGY/INFORMATION BIT

\begin{tabular}{lcc}
\hline Pulse Rate & P & 100 per Second \\
Sigral-to-Noise & $\frac{\mathrm{S}}{\mathrm{N}}$ & $20(13 \mathrm{~dB})$ \\
Transmutter Power & $\mathbf{P}_{\mathrm{s}}$ & $1(0 \mathrm{dBW})$ \\
Binary Word & $\mathrm{I}$ & $1(0 \mathrm{~dB})$ \\
Transmitter Optical & $\mathrm{L}^{\prime \prime}$ & $1(0 \mathrm{~dB})$ \\
Attenuation & & $1(0 \mathrm{~dB})$ \\
Quantum Eificiency & $\eta$ & $1(0 \mathrm{~dB})$ \\
\hline
\end{tabular}

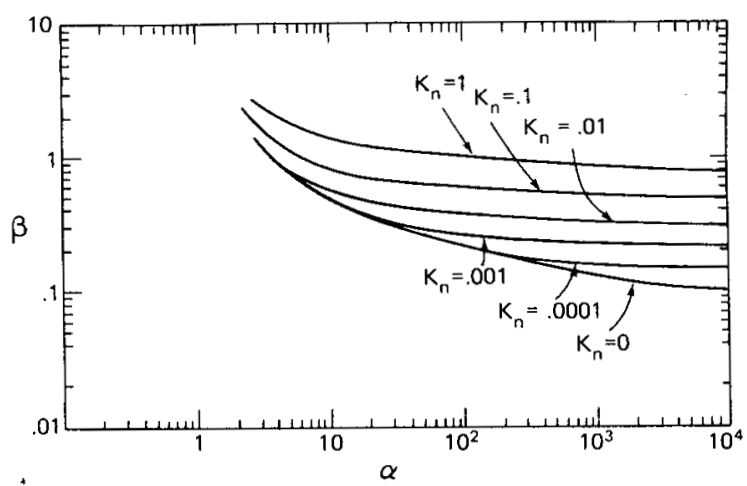

Fig. 3. Theoretical $\beta$-efficiency of wide-band PPM systems with additive noise as a function of $\alpha$.

$\alpha=B_{s} / H$ for different values of $K_{n}$ [9]. The asymptotic limit is [9]

$$
\beta_{\text {asym }}=\frac{1}{\log \left(1+\frac{1}{K_{n}}\right)} .
$$

\section{SYSTEM DESIGN}

We are now in a position to solve for the available margin $L$ by equating

$$
\begin{aligned}
K_{s} & =\frac{\eta E}{h f} L L^{\prime} \\
& =\frac{1}{2} \frac{S}{N}\left[1+\sqrt{1+\frac{4 K_{n}}{(S / N)}}\right] \\
& =\frac{1}{2} \frac{S}{N}\left[1+\sqrt{1+\frac{4 \eta N(f) B_{s} \Delta T \Omega_{f o v} A L}{(S / N) h f}}\right]
\end{aligned}
$$

using (4) and (13). We make one simplification by assuming that the worst case will be on a cloudy day. Then the solar (or lunar) energy entering the water is diffused, and we can replace the spectral radiance by the spectral irradiance $W(f) / \pi$ $\mathrm{W} / \mathrm{M}^{2} / \mu \mathrm{m}$. Recognizing that the pulse energy per bit $E=P_{s} / P$ where $P_{s}$ is the transmitter power, we have

$$
\frac{\Omega_{f o \nu \eta P_{s} L L^{\prime}}}{I h f P}=\frac{1}{2}\left(\frac{S}{N}\right)\left[1+\sqrt{1+\frac{4_{\eta} W(f) B_{s} \Delta T \Omega_{f o v} A L}{I \pi(S / N) h f}}\right] .
$$

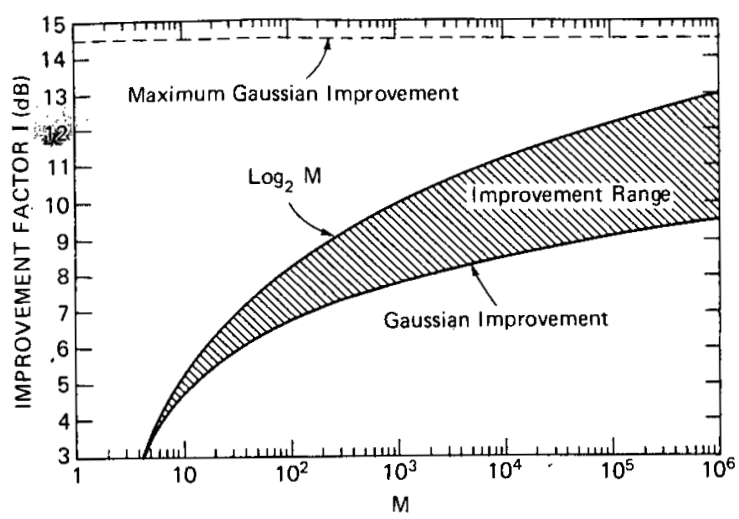

Fig. 4. Performance improvement using $M$-ary signaling.

We have included the improvement factor $I$ to indicate the increase in bits per symbol achieved by an $M$-ary alphabet and $S / N$ is now the signal-to-noise ratio per bit. We have plotted $I$ in Fig. 4 for Gaussian statistics and $P_{E}=10^{-6}$. We also consider $L^{\prime}$ as a product of the geometric spread and the transmitter optics losses $L^{\prime \prime}$ so that

$$
L^{\prime}=\frac{L^{\prime \prime} A}{\frac{\pi}{4} D^{2}}
$$

where $D$ is the projected spot diameter. Finally, we assume that the field of view of the signal is $1 \mathrm{sr}$ and signal power is lost if $\Omega_{\mathrm{fov}}<1$. Thus, the received power is $\Omega_{\mathrm{fov}} P_{s}$. We consider two conditions.

1) Quantum Limited:

$$
\begin{aligned}
& \frac{\Omega_{f o v} P_{s} L L^{\prime \prime} A}{\operatorname{IhfP} \frac{\pi}{4} D^{2}}=\frac{S}{N} \\
& L=\left(\frac{S}{N}\right) \frac{h f P \frac{\pi}{4} D^{2} I}{\eta P_{s} L^{\prime \prime} A \frac{\pi}{4} \Omega_{f o v}} .
\end{aligned}
$$

2) Background Limited:

$$
\begin{aligned}
& \frac{\Omega_{f o \nu} \eta P_{s} L L^{\prime \prime} A}{h f P \frac{\pi}{4} D^{2}}=\sqrt{\frac{\eta W(f) B_{s} \Delta T \Omega_{f o v} A L(S / N) I}{\pi h f}} \\
& L=\left(\frac{P D^{2}}{4 P_{s} L^{\prime \prime}}\right)^{2} \frac{\pi W(f) h f\left(B_{s} \Delta T / \Omega_{f o v}\right) S / N I}{\eta A}
\end{aligned}
$$

In order to have general applicability, we can create a standard design curve by normalizing. Thus, we assume a standard receiver to have the attributes in Table II.

We then designate the link loss as $-10 \log _{10} L$ and we use the following units:

$D=$ spot size in kilometers

$T=$ pulse width in microseconds

$B_{s}=$ optical bandwidth in angstroms.

We then obtain a link loss in decibels as follows. 
TABLE II

PARAMETERS FOR THE STANDARD RECEIVER

\begin{tabular}{ccc}
\hline$K_{s}$ & $M$ & $K_{s} / C$ \\
\hline 0.8 & 19 & 0.593 \\
0.9 & 23 & 0.552 \\
1.0 & 28 & 0.506 \\
1.2 & 35 & 0.494 \\
1.4 & 42 & 0.484 \\
1.6 & 47 & 0.488 \\
1.8 & 52 & 0.496 \\
2.0 & 55 & 0.508 \\
5.0 & 95 & 0.792 \\
\hline
\end{tabular}

1) Quantum Limited:

$$
\begin{aligned}
-10 \log _{10} L= & -10 \log _{10}\left(\frac{S}{N}\right)-10 \log _{10} P-20 \log _{10} D \\
& +10 \log _{10} \eta+10 \log _{10} P_{s}-10 \log _{10} I \\
& +10 \log _{10} L^{\prime \prime}+10 \log _{10} A \\
& +10 \log _{10} \Omega_{f o v}+124 .
\end{aligned}
$$

2) Background Limited (Daytime)-W(f) $=1.45 \times 10^{3}$ $\mathrm{W} / \mu \mathrm{m} \cdot \mathrm{M}^{2}$ :

$$
\begin{aligned}
-10 \log _{10} L= & -20 \log _{10} P-40 \log _{10} D-10 \log _{10} \rho \\
& -10 \log _{10}(S / N)+20 \log _{10} P_{s}-10 \log _{10} I \\
& +20 \log _{10} L^{\prime \prime}+10 \log _{10} \eta \\
& +10 \log _{10} A+139.5
\end{aligned}
$$

3) Background Limited (Nighttime)-W(f) $=3 \times 10^{-3}$ $\mathrm{W} / \mu \mathrm{m} \cdot \mathrm{M}^{2}$ :

$$
\begin{aligned}
-10 \log _{10} L= & -20 \log _{10} P-40 \log _{10} D-10 \log _{10} P \\
& -10 \log _{10}(S / N)+20 \log _{10} P_{s}-10 \log _{10} I \\
& +20 \log _{10} L^{\prime \prime}+10 \log _{10} \eta \\
& +10 \log _{10} A+199.5
\end{aligned}
$$

where we use

$$
\rho=\frac{\Delta T B_{s}}{\Omega_{f o v}} .
$$

The standard curves then divide by day and night conditions (Figs. 5 and 6). To compute the performance of another system, each item would be assessed and a margin computed.

As an example, consider a system requiring a $50 \mathrm{~dB}$ margin for clouds and water. It has the characteristics in Table III.

Plotting positive net loss up (and negative net loss down) and crossing over to the intersection (hatched lines) in Figs. 5 and 6 , we see that in daytime, a $37 \mathrm{~dB}$ margin intersects the $\rho=10$ curve at a smaller value of $D$ than where $57 \mathrm{~dB}$ intercepts the Q.L. curve. This occurs at $D=8 \mathrm{~km}$ and is the zero margin spot size for the system. Similarly, at night we are background limited, but at $D \sim 200 \mathrm{~km}$. On the other hand, if

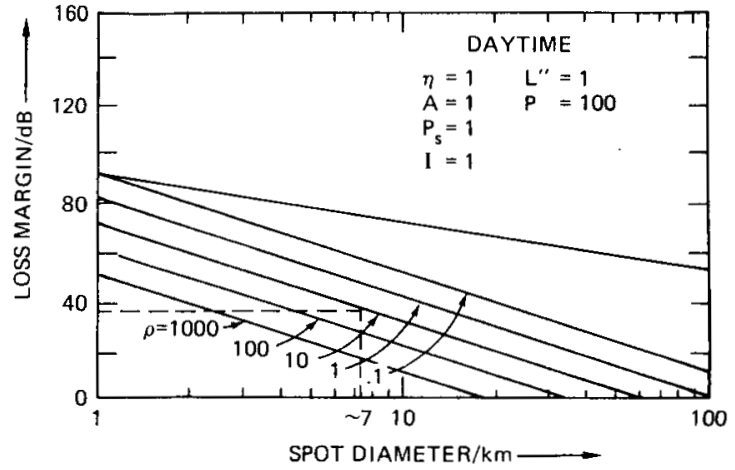

Fig. 5. Standard performance curve-day time.

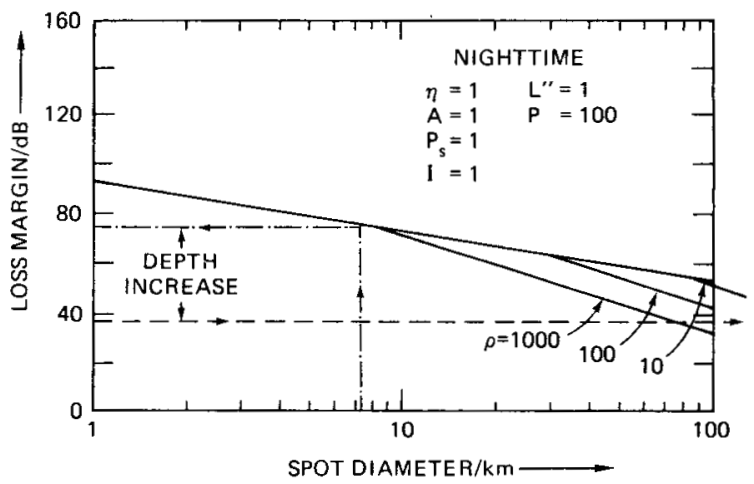

Fig. 6. Standard performance curve-nighttime.

TABLE III EXAMPLE SYSTEM DESIGN

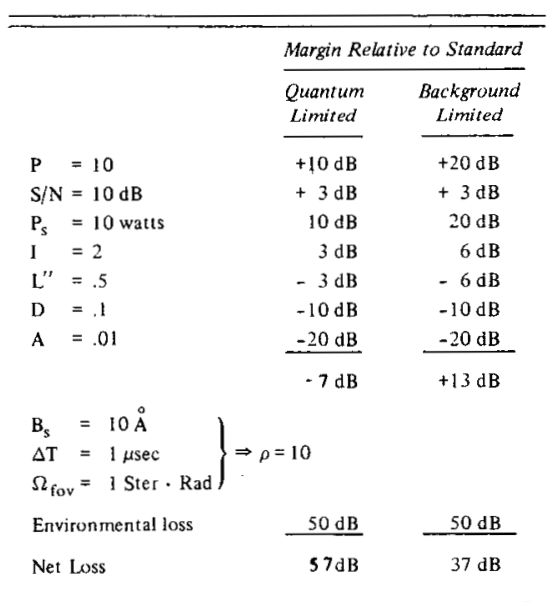

we choose to operate at $D=8 \mathrm{~km}$ at night, we move vertically up from $D=8 \mathrm{~km}$ and reach the merged $\rho=10$ and Q.L. curves at a margin of $75 \mathrm{~dB}$, which means that we would have an additional $36 \mathrm{~dB}$ to use, i.e., we could operate deeper at night with an $8 \mathrm{~km}$ spot size.

The environmental loss is composed of cloud loss and water loss. A detailed assessment of these effects is beyond the scope of this paper. However, the gross effects can be determined by using the van de Hulst equation for cloud loss:

$$
L_{\text {cloud }} \cong \frac{1}{1.42+(0.125) \tau}
$$


where $\tau$ is the optical thickness of the cloud, the real thickness multiplied by the cloud extinction coefficient. Typically, $\tau$ can range from 10 to 200 so that the cloud loss can range from 4 to $14 \mathrm{~dB}$. The water loss is gerierally taken to be

$$
L_{\text {water }}=e^{-K Z}=e^{-\tau_{d}}
$$

where $K$ is the diffuse attenuation, $Z$ is the depth, and $K Z=$ $\tau_{d}$ is the optical diffusion thickness of the water. Typically, $\tau_{d}$ can range from 1 to 10 so that the water loss can range from 5 to $50 \mathrm{~dB}$, dependent upon the actual depth and the particular water type.

\section{SUMMARY}

In this paper we have motivated a desire to communicate to submerged SSBN's without the latter deploying appendages in any form. We next outlined an optical communication system operating in the Blue/Green portion of the EM spectrum that offers the potential for accomplishing this result. This system is currently in the R \& D phase of DoD activity and is slowly evolving a design philosophy. Our primary objective has been to expose this design philosophy and to explore some of the attendant technical issues. While the environmental difficulties have only been addressed briefly, the numbers used are representative of what can be expected.

\section{REFERENCES}

[1] Naval Electron. Lab. Cen., San Diego, CA, Tech. Document 490 , NELC/TD 490, June 1976.

[2] R. M. Gagliardi and S. Karp, Optical Communications. New York: Wiley-Interscience, 1976.

[3] Proc. IEEE, Special Issue on Optical Communications, vol. 58, Oct. 1970.

[4] S. Karp and J. R. Clark, "Photon counting: A problem in classical noise theory," IEEE Trans. Inform. Theory, vol. IT-16, Nov. 1970.

[5] R. M. Gagliardi and S. Karp, " $M$-ary Poisson detection and optical communications,' IEEE Trans. Commuñ. Technol., vol. COM-17, Apr. 1969.

[6] A. J. Viterbi, Principles of Coherent Communications. New York: McGraw-Hill, 1966

[7] R. M. Fano, Transmission of Information. Cambridge, MA M.I.T. Press and New York: Wiley, 1961.

[8] S. Karp and R. M. Gagliardi, "The design of a pulse position modulated optical communication system," IEEE Trans. Commun. Technol., vol. COM-17, Dec. 1969
[9] S. Karp, "Communication efficiency of quantum systems,"' NASA Tech. Rep. NASA TR R-320, Sept. 1969.

[10] Naval Ocean Syst. Cen., San Diego, CA, Tech. Note 279, NOSC TN 279, G, Lee. C. Ciany, G. Schroeder, and J. Fenier, Appendix B, "Availability models for space-to-earth optical communication links."

[11] S. Q. Duntley, "Underwater lighting by submerged lasers and incandescent sources," USCD Visibility Lab., SIO Ref. 71-1, Final Rep., June 1971.

[12] S. Karp, "Optical communications between underwater and above surface (satellite) terminals," IEEE Trans. Commun., vol. COM24, Jan. 1976

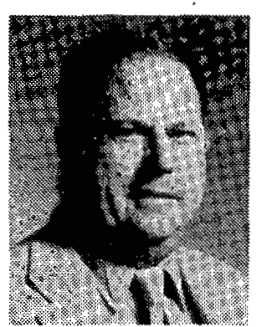

Thomas F. Wiener ('S'55-M'62) received the Sc.B.(E) degree from Brown University, Providence, RI, in 1958 and the Sc.D. degree from the Massachusetts Institute of Technology, Cambridge, where his dissertation dealt with strapdown inertial navigation systems.

$\mathrm{He}$ has served in nuclear-powered ballistic missile submarines, and commanded the USS JACK(SSN605). He is now a Program Manager at the Defense Advanced Research Projects Agency, Arlington, VA, where his responsibilities include laser communications, cruise missile guidance, advanced submarines, and surveillance systems. He has authored several papers and chaired several technical symposia in his fields of interest.

Dr. Wiener is a member of Tau Beta Pi, Sigma Gamma Tau, and Sigma $\mathrm{Xi}$.

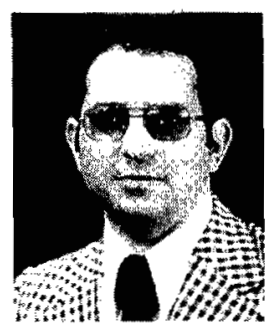

Sherman Karp (M'62) received the B.S.E.E. and M.S.E.E. degrees from the Massáchusetts Institute of Technology, Cambridge, in 1960 and 1962, respectively, and the Ph.D. degree from the University of Southern California, Los Angeles, in 1967.

He has done extensive research in electromagnetic systems. Currently is a Program Manager in the Strategic Technology Office of DARPA, Arlington, VA, where he is involved in the development of advanced systems and technology. He is a coauthor of Optical Conmunications (New York: Wiley, 1976) and Optical Transmission (New York: Plenum, to be published) and co-Guest-Editor of two Special Issues of the IEEE TRANSACTIONS ON COMMUNICATIONS on Optical Communications and Aeronautical Communications.

Dr. Karp is a member of Tau Beta Pi, Eta Kappa Nu, and Sigma Xi. 